\title{
Serial Organization of Human Behavior in the Inferior Parietal Cortex
}

\author{
Thomas Jubault, Chrystele Ody, and Etienne Koechlin \\ Inserm, Département d’Etudes Cognitives, Ecole Normale Supérieure, Université Pierre et Marie Curie, 75005 Paris, France
}

The parietal cortex is involved in a wide range of cognitive functions in humans including associative functions between multiple sensorimotor spaces, attentional control, and working memory. Little is known, however, about the role and the functional organization of the parietal cortex in action planning and sequential cognition. Moreover, the respective contributions of parietal and frontal regions to action planning remains poorly understood. To address this issue, we designed a functional magnetic resonance imaging protocol requiring subjects to perform overlearned sequences of motor acts and sequences of cognitive tasks. The results reveal only a single bilateral region in the cerebral cortex located in the intraparietal sulcus (IPS; Brodmann's area 40) exhibiting sustained activations during the execution of both motor and task sequences. Additional analyses of phasic activations during sequence execution further suggest a functional dissociation between the left IPS, involved in representing and processing the abstract serial structure of ongoing behavioral sequences regardless of their hierarchical structure, and the right IPS, involved in preparing successive sensorimotor sets that compose such behavioral sequences. We show that this parietal system functionally differs from the frontal system that was previously identified as controlling action selection with respect to the hierarchical rather than serial structure of behavioral plans. Thus, our results reveal the central role of the bilateral intraparietal sulcus in high-order sequential cognition and suggest a major functional segregation within the frontoparietal network mediating action planning, with the frontal and parietal sector involved in processing the hierarchical and serial structure of action plans, respectively.

Key words: humans; fMRI; parietal cortex; behavioral sequences; planning; cognitive control

\section{Introduction}

The frontal-parietal network forms a critical central system for the organization of goal-directed behavior (Fuster, 1989; Barash, 2003). Frontal regions including premotor and prefrontal regions most often coactivate with the inferior parietal lobules (IPLs) [e.g., during action planning (Baker et al., 1996; Fincham et al., 2002) and cognitive set-shifting (Asari et al., 2005; Collette et al., 2005; Shafritz et al., 2005)]. Specifically, the IPL, besides its involvement in many cognitive functions including perceptual attention and polymodal associative processes (for review, see Andersen et al., 1997; Culham and Kanwisher, 2001; Cohen and Andersen, 2002), is engaged in various aspects of action planning such as encoding (Koechlin et al., 2002; Sakai et al., 2002), storage (Wu et al., 2004), execution (Sadato et al., 1996; Catalan et al., 1998; Bengtsson et al., 2004), monitoring (Deiber et al., 1996), and representations of behavioral sequences (Grafton et al., 1998; Behrmann et al., 2004; Bengtsson et al., 2005). However, although the IPL involvement in action planning is well substanti-

Received Jan. 10, 2007; revised June 28, 2007; accepted June 29, 2007.

This work was supported by the French Ministry of Research (ACI number 22-2002-350) and the European Young Investigator Award (to E.K.). We thank Jean-Luc Anton, Bruno Nazarian, and Muriel Roth at the Magnetic Resonance Imaging (enter in Hospital La Timone (Marseille, France) for MRI facilities and technical assistance.

Correspondence should be addressed to Dr. Etienne Koechlin, Inserm U742, Université Pierre et Marie Curie, 9, quai St. Bernard, 75005 Paris, France. E-mail: koechlin@ccr.jussieu.fr.

D0I:10.1523/JNEUROSCI.1986-07.2007

Copyright $\odot 2007$ Society for Neuroscience $\quad 0270-6474 / 07 / 2711028-09 \$ 15.00 / 0$ ated, the respective contributions of the IPL and frontal regions to action planning remains poorly understood.

To address this issue, we used functional magnetic resonance imaging (fMRI) to examine both sustained and phasic brain activations, while subjects were executing prelearned behavioral sequences including sequences of motor acts and sequences of cognitive tasks. The motor sequence consisted of generating fixed sequences of motor responses successively triggered by visual stimuli, whereas the task sequence consisted of performing fixed sequences of basic categorization tasks generating motor responses contingent on visual stimuli. Accordingly, these behavioral sequences were designed to independently vary the serial and hierarchical structure of action plans. In a previous study (Koechlin and Jubault, 2006), we analyzed activations in the frontal lobes. We showed that lateral frontal regions exhibited a pattern of phasic activations indicating that lateral frontal regions are organized to select action segments according to the hierarchical structure of behavioral sequences and regardless of their serial structure. Assuming that the IPL subserves a complementary function, this finding also suggests that the IPL, in contrast, is organized to process the serial rather than hierarchical structure of action plans. The hypothesis states that the IPL keeps track of serial progression and represents serial associations guiding the sequential execution of action plans. In the present study, we analyzed posterior brain activations to test this hypothesis. More specifically, the hypothesis predicts that during the execution of behavioral sequences, IPL regions exhibit sustained activations 
combined with differential phasic activations corresponding to sequence increments and boundaries, regardless of the hierarchical structure of behavioral sequences (sequences of motor acts vs sequences of cognitive tasks). Alternative hypotheses would be that distinct IPL regions are involved in processing the serial structure of action plans according to their hierarchical structure or that functional segregations between parietal and frontal regions are unrelated to the distinction between serial versus hierarchical processing underlying action planning.

\section{Materials and Methods \\ Experimental protocol}

The data presented here were collected in the experiment described by Koechlin and Jubault (2006). This fMRI experiment included 16 subjects (right handed; age, 22-28 years), who provided written informed consent approved by the French National Ethics Committee. A few days before scanning sessions, subjects received written instructions and informally performed the task with experimenter feedback. Then, subjects were trained and executed 56 times each of the two behavioral sequences subsequently used in the scanner (a motor sequence and a sequence of cognitive tasks; see below). As confirmed by behavioral results reported previously (Koechlin and Jubault, 2006), all behavioral sequences were overlearned by the time subjects entered the scanner. Subjects received no feedback related to their performance in the scanner.

The protocol included two experimental conditions referred to as motor and task conditions, which were administered in separate scanning sessions. In the motor condition, subjects executed sequences of single motor acts; in the task condition, they performed sequences of categorization tasks. The order of conditions was counterbalanced across subjects. In each condition, the subject had to press hand-held left or right buttons in response to successive visual stimuli, alternating periods when they performed a baseline task and periods when they executed the prelearned behavioral sequence (Fig. 1). Subjects stopped executing the baseline task and started executing the behavioral sequence when a start cue occurred (stimulus color switched to green). Then, when a stop cue occurred (stimulus color switched to red), subjects stopped executing the sequence and resumed on the baseline task.

In the motor condition, stimuli were colored squares. In the baseline periods, subjects repeatedly pressed both buttons simultaneously (left and right) in response to each stimulus. In sequence execution periods, the subject executed the prelearned sequences of button presses in response to successive stimuli, which was as follows: left and right $>$ left and right $>$ right $>$ right $>$ left.

In the task condition, stimuli were pseudorandomly chosen letters A, $\mathrm{B}$, or C. In the baseline periods, subjects had to detect occurrences of letter A by pressing the right button if the presented letter was an A and the left button otherwise. This sensorimotor mapping or task set is referred to as $\mathrm{TS}_{\mathrm{A}}$. In the sequence execution periods, subjects executed the following prelearned sequence of task sets: $\mathrm{TS}_{\mathrm{A}}>\mathrm{TS}_{\mathrm{A}}>\mathrm{TS}_{\mathrm{B}}>\mathrm{TS}_{\mathrm{B}}>$ $\mathrm{TS}_{\mathrm{C}}$.

Task sets $\mathrm{TS}_{\mathrm{A}}, \mathrm{TS}_{\mathrm{B}}$, and $\mathrm{TS}_{\mathrm{C}}$ corresponded to the following sensorimotor mappings: $\mathrm{TS}_{\mathrm{A}},(\mathrm{A}>$ right, $\mathrm{B}>$ left, $\mathrm{C}>$ left $)$; $\mathrm{TS}_{\mathrm{B}},(\mathrm{A}>$ left, $\mathrm{B}>$ right, $\mathrm{C}>$ left $) ; \mathrm{TS}_{\mathrm{C}},(\mathrm{A}>$ left, $\mathrm{B}>$ left, $\mathrm{C}>$ right). Letters $\mathrm{A}, \mathrm{B}$, and $\mathrm{C}$ were pseudorandomized so that the proportion of left and right responses was equal.

\section{Stimuli contingency and timing}

In both conditions, stop cues occurred four trials (at the end of learned sequences; endogenous termination) or three, two, or one trials (exogenous terminations) after start cues with the following proportions: 49 , 17,17 , and $17 \%$, respectively. Importantly, start cues occurred after stop cues with exactly the same contingencies, so that series of stimuli composing baseline and sequence execution periods had the same length in average. Stimuli duration was $500 \mathrm{~ms}$. Stimulus onset asynchronies were varied, ranging from 2400,4800 , and $7200-9600 \mathrm{~ms}$ to dissociate eventrelated hemodynamic responses to each trial and sustained activations during sequence execution. Timing of events was the same in both the motor and task conditions as well as in baseline and sequence execution

\section{A Experimental design}

\section{Task condition}

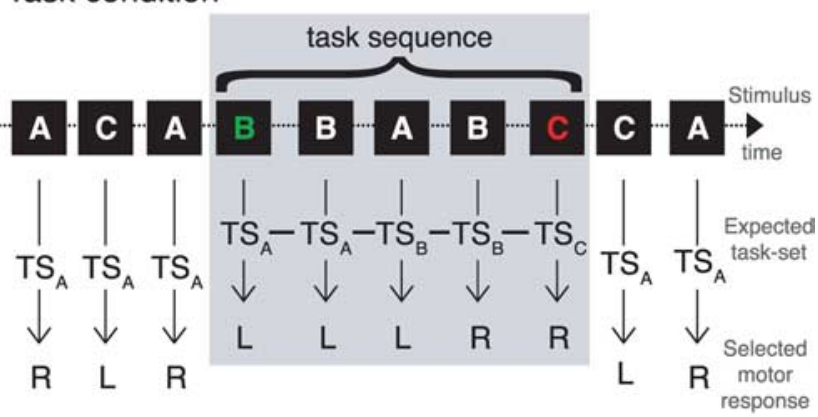

\section{Motor condition}

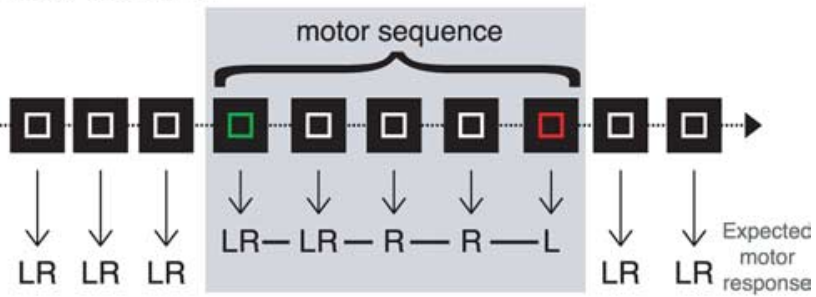

\section{B Serial events}

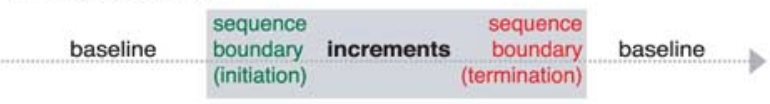

\section{Hierarchical nesting}



Figure 1. Experimental protocol. $\boldsymbol{A}$, Truncated series of successively presented visual stimuli are shown in both the motor and task condition. Green and red cues were start and stop signals, respectively. In the motor condition, stimuli were square symbols. In each trial, subjects had to select left $(L)$, right $(R)$, or simultaneous left and right $(L R)$ finger presses (as indicated by arrows). In the task condition, stimuli were pseudorandomly chosen letters $A, B$, or $C$. In each trial, subjects had to select the sensorimotor task set $\left(\mathrm{TS}_{A}, \mathrm{TS}_{B}\right.$, or $\mathrm{TS}_{\mathrm{C}}$ as indicated by arrows) to produce the correct motor response to stimuli (indicated below each expected task sets). Examples of endogenous terminations are shown. $\boldsymbol{B}$, Serial organization of behavioral events in both conditions. C, Hierarchical organization of behavioral sequences in the motor and task condition. See Material and Methods for terminology and additional details.

periods. Finally, to avoid possible concatenations of overlearned sequences with subsequent baseline trials into longer sequences, stop cues were actually replaced by start cues in half of the termination trials, indicating subjects to stop and restart the sequence from the beginning. Restart, initiation, and termination trials were associated with virtually the same brain activations. Therefore, we factored out restart trials in all reported analyses for clarity. For simplicity, we referred to initiation and termination trials as sequence boundaries and to intermediate trials between start and stop cues as increments. 
Serial versus hierarchical organization of action sequences

The motor and task sequences described above have the same serial structure but involve distinct levels in the hierarchical organization of action. According to Koechlin and Jubault (2006), the motor sequence (motor condition) and task sets $\mathrm{TS}_{\mathrm{A}}, \mathrm{TS}_{\mathrm{B}}$, and $\mathrm{TS}_{\mathrm{C}}$ correspond to simple action chunks [i.e., sequential or parallel sets of single motor acts (or sensorimotor associations)]. In contrast, the task sequence composed of task sets $\mathrm{TS}_{\mathrm{A}}, \mathrm{TS}_{\mathrm{B}}$, and $\mathrm{TS}_{\mathrm{C}}$ corresponds to a superordinate action chunk (i.e., a chunk of simple action chunks). Single motor acts and simple and superordinate action chunks are three nested levels in the hierarchical organization of action. The motor sequence is a simple chunk and involves sequential associations between single acts. In contrast, the task sequence is a superordinate chunk and involves sequential associations between simple chunks.

\section{Predictions}

For simplicity, we referred to increased phasic activations at sequence boundaries compared with increments as effects of sequence boundaries. Similarly, we referred to increased activations in increments compared with baseline trials as effects of increments. Using the protocol described here, we previously showed that the lateral frontal cortex is engaged in processing the hierarchical structures of action chunks, regardless of their serial organization (Koechlin and Jubault, 2006). Indeed, (1) no sustained activations during motor and task sequence execution were observed in lateral frontal regions; (2) lateral premotor regions were the only frontal regions that exhibited effects of motor increments (selection/inhibition of single motor acts); (3) posterior prefrontal regions [Brodmann's area (BA) 44] showed no effects of motor increments but showed effects of task increments and motor sequences boundaries (i.e., effects associated with selection/inhibition of simple action chunks only, regardless of their serial organization); and (4) slightly more anterior prefrontal regions (BA 45) exhibited effects of task sequence boundaries only (i.e., effects associated with selection/inhibition of superordinate chunks only).

In the present study, we investigated whether the IPL subserves the complementary function, namely the processing of serial structures of action chunks, regardless of their hierarchical organization. This hypothesis states that the IPL keeps track of sequential progression and represents sequential associations that are required to move on along serial structures regardless of hierarchical levels. More specifically, the hypothesis predicts that IPL regions exhibit sustained activations from sequence initiation to termination in both motor and task sequences. Furthermore, such IPL regions are expected to show phasic activations whenever subjects moved on in both motor and task sequences and should be especially sensitive to sequence boundaries when subjects load and inhibit such sequence representations. Thus, we especially examined whether IPL regions jointly exhibited (1) sustained activations during the execution of both motor and task sequences compared with baseline periods; (2) phasic activations in motor and task increments compared with baseline trials; and (3) increased phasic activations at motor and task sequence boundaries compared with increments.

\section{Data acquisition}

The motor and task conditions were administered in separate scanning sessions on separate days. Each scanning session was preceded by a training session (see above). The order of conditions was counterbalanced across subjects. In each scanning session, a 3T Brucker whole-body and radio frequency coil scanner was used to perform first an anatomical scan and then six series of 247 functional scans for each subject (repetition time, $2 \mathrm{~s}$; echo time, $35 \mathrm{~ms}$; field of view, $192 \times 192 \mathrm{~mm}^{2}$; acquisition matrix, $64 \times 64$; flip angle, $78^{\circ}$; 24 interleaved and jointed slices; voxel size, $3 \times 3 \times 5 \mathrm{~mm}$ ). In those six series, sequences were executed 84 times. There were, consequently, 84 baseline periods, 84 initiation trials, and 84 termination trials. Forty-two terminations were endogenous, and 42 were exogenous. There were 168 increment trials in sequences and 168 trials in baseline periods.

The experimental protocol was administered using the Labview software package. fMRI data were processed using the SPM99 software package (http://www.fil.ion.ucl.ac.uk/spm/) with standard interslice tempo- ral reslicing, spatial realignment, linear normalization to the Talairach and Tournoux (1988) stereotaxic atlas (Hôpital La Timone-Marseille template), and spatial (isotropic three-dimensional Gaussian kernel, 10 $\mathrm{mm}$ ) and temporal (Gaussian kernel, $4000 \mathrm{~ms}$ ) smoothing.

\section{Data analyses}

All data analyses and statistical contrasts were the same as those reported previously by Koechlin and Jubault (2006).

Computation of brain activations. We computed statistical parametric maps from local fMRI signals using a linear multiple regression model. The model included three sets of regressors for each condition. (1) Eventrelated regressors were impulse functions convolved by the canonical hemodynamic response function. Those regressors separately modeled baseline trials, sequence initiation trials (trials including start cues), increment trials (intermediate trials occurring after start cues and before stop cues), endogenous and exogenous sequence termination trials (trials including stop cues), and error trials. (2) Epoch-related regressors were variable-length boxcar functions convolved by the canonical hemodynamic response function, separately modeling sustained effects associated with series of baseline and sequence trials delimited by start and stop cues. (3) Scan-related regressor modeling scanning series and signal drifts included constant, linear, and quadratic functions.

Activations were first identified using a fixed-effect model assessing the fit between the multiple regression model described above and time courses of local magnetic resonance signals (voxelwise threshold: $Z>$ $4.3, p<0.05$ corrected for multiple comparisons over the whole brain; extent threshold: $p<0.05,832 \mathrm{~mm}^{3}$ ). Then, to account for betweensubjects variability and to allow inferences at the population level, regional activations identified above were subsequently assessed using a random-effect model (voxelwise threshold: $p<0.05$ corrected for multiple comparisons over the search volumes). Additionally, joint activations across both conditions were computed by masking activations separately obtained in each condition using uncorrected significance thresholds (fixed-effects model: $Z>3.1, p<0.001$ uncorrected; random-effect model: $p<0.01$ uncorrected).

ANOVAs. In each region identified above, we also computed the mean event-related hemodynamic response (mER-HRs) related to each trial type from the peristimulus fMRI signal observed in each voxel, first by subtracting the estimated contribution of other events based on parameter estimates of the multiple linear regression model and then by averaging the resulting responses over each activation cluster. Peaks of mERHRs (i.e., maximal signal changes) in identified regions were then entered in separate repeated-measure ANOVAs with conditions and trial types as within-subject factors. In a first ANOVA, the trial-type factor contrasted sequence boundary versus increment trials for assessing effects of sequence boundary. In a second and third ANOVA, the trial-type factor contrasted initiation versus termination trials and endogenous versus exogenous termination trials, respectively. Finally, in a fourth ANOVA, the trial-type factor contrasted increments versus baseline trials for assessing effects of successive selection of sequence components.

\section{Results}

\section{Behavioral results}

Behavioral results were reported by Koechlin and Jubault (2006). Briefly, behavioral performances in both conditions indicate that no residual learning occurred during scanning. In each condition, error rates were lower than $5 \%$ in both baseline and sequence execution periods. Behavioral reaction times (RTs) were larger in initiation/termination trials than intermediate trials of behavioral sequences (all $F$ values $>4.6$; $p<0.048$ ). In both conditions, no significant differences in RTs were observed between initiation and termination trials, as well as between termination trials occurring at the end of learned sequences (endogenous terminations) and those occurring earlier (exogenous terminations) (all $F$ values $<1$ ). 




Figure 2. Topography of brain activations. Purple indicates regions exhibiting sustained activations during motor and task sequences. Blue indicates regions exhibiting sustained activations during motor sequences only. Green and yellow indicate regions showing all phasic effects [i.e., effects of motor and task increment, motor and task sequence boundary (green, larger effect of motor increment; yellow, larger effect of motor sequence boundaries)]. All these phasic effects were also observed in the left purple region. Orange indicates regions exhibiting effects of motor increment, motor sequence boundary, and task increment but no effect of task sequence boundary. Joint effects of motor sequence boundary and task increment were also observed in the blue and right purple regions. Red indicates regions exhibiting only effects of initiation and endogenous termination in motor and task sequences. Activations are superimposed on anatomical axial slices averaged across subjects (neurological convention) and indexed by the vertical Talairach coordinate ( $z$ ). Frontal activations are not shown here [reported by Koechlin and Jubault (2006)]. Talairach coordinates of activation peaks are provided in Tables 1 and 2. L, Left; R, right.

\section{fMRI results}

Consistently with behavioral performance, we observed virtually no significant differences in statistical parametric maps among initiation, endogenous, and exogenous termination trials composing motor and task sequences. Consequently, those events were pooled together and referred to as motor and task sequence boundaries, respectively. Also, for clarity we refer to intermediate trials in motor and task sequences as motor and task increments, respectively. These events corresponded to successive selection of motor responses and task sets composing motor and task sequences, respectively. We report here only activations located in the posterior part of the cerebral cortex (i.e., in regions posterior to the central sulcus). Frontal activations were reported in a previous study (Koechlin and Jubault, 2006).

\section{Sustained activations}

We first computed regions exhibiting sustained activations during the execution of motor and task sequences compared with baseline periods. This analysis revealed a bilateral region located in the intraparietal sulcus (IPS; BA 40) showing sustained activations during the execution of both motor and task sequences (Table 1, Figs. 2, purple region, $3 A$ ). No other regions were found to exhibit sustained activations, except a more ventral region located in the right IPS that showed sustained activations during execution of motor sequences only (Table 1, Figs. 2, blue region, $3 A)$.

\section{Phasic activations}

To describe phasic activations, we referred to increased activations at sequence boundaries compared with increments as ef- fects of sequence boundaries. Similarly, we referred to increased activations in increments compared with baseline trials as effects of increments.

In the left hemisphere, we observed significant phasic activations in two regions located in the anterior and posterior IPS (Table 2, Fig. 2, yellow and green regions). Both regions exhibited effects of motor and task sequence boundaries as well as effects of motor and task increments (Fig. $4)$. Both regions were contiguous to the tonically activated IPS region described above. Post hoc analyses revealed that in this latter region, all the phasic effects were also significant (see below and Fig. 3B).

In the right hemisphere, significant phasic activations were observed in a single region located in the middle IPS (Table 2, Fig. 2, orange region). In this region, we found significant effects of motor sequence boundaries and task increments. Additional post hoc analyses also revealed effects of motor increment in this region (see below and Fig. 4). The region was contiguous to the two ventral and dorsal IPS regions showing sustained activations. Again, post hoc analyses revealed that in these two IPL regions, effects of motor sequence boundaries and task increments were significant (see below and Fig. 3B).

In addition, phasic activations were found in two bilateral regions. First, significant effects of task increment were observed in the precuneus (BA 7) (Table 2,

Fig. 2, orange region). Post hoc analyses also revealed that precuneus activations were similar to those in the right IPL region (see below and Fig. 4). Second, significant effects of task sequence boundaries were observed at the inferior, anterior part of the supramarginal gyrus [BA 40; supramarginal gyrus at the temporoparietal junction (SMG/TPJ)] (Table 2, Fig. 2, red regions). Post hoc analyses also indicated that this region responded to initiation and endogenous termination of both motor and task sequences (see below and Fig. 5).

\section{Post hoc factorial analyses}

Post hoc factorial ANOVAs were conducted in regions identified above by averaging magnetic resonance signals over the corresponding activation clusters (see Materials and Methods). Such analyses allow to possibly detect additional joint effects in these regions by minimizing type II statistical errors.

First of all, in both conditions, the analyses confirmed the absence of differential phasic activations at sequence boundaries between initiation and termination (all $F$ values $<1.9$; all $p$ values $>0.18$ ). There was only a marginal effect of motor sequence termination compared with initiation in the right ventral IPL region exhibiting sustained activations in motor sequences (Fig. 2, blue region) $(F=4.3 ; p=0.06)$. Also, the analyses confirmed similar phasic activations in endogenous and exogenous termination in all regions described above (all $F$ values $<2.4$; all $p$ values $>0.14$ ), except in the bilateral SMG/TPJ region (see below and Fig. 2, red regions). 
Left parietal regions

The ANOVAs also confirmed that all left parietal regions identified above, except the SMG/TPJ region, showed significant effects of motor and task increments as well as motor and task sequence boundaries. In particular, such effects were found in the left middle IPS region showing sustained activations (all $F$ values $>9.5$; all $p$ values $<0.008$ ). Additionally, effects of motor increments and motor sequence boundaries differed between the anterior and posterior left IPS regions exhibiting only phasic activations. The effect of motor increment was larger in the anterior than posterior left IPS regions (regions $X$ motor increment: $F=11.8 ; p=0.004$ ), and consistently, the effect of motor sequence boundaries showed the converse interaction (regions $\times$ motor sequence boundaries: $F=4.73$; $p=0.046)$.

\section{Right parietal regions}

In the right parietal cortex, the ANOVAs revealed that the middle IPS region exhibiting only phasic activations showed significant effects of motor and task increments, effects of motor sequence boundaries (all $F$ values $>15.6 ; p<$ $0.0013)$, but no effects of task sequence boundaries $(F=1.7 ; p=0.21)$. The precuneus showed the same pattern of results with effects of motor and task increments (both $F$ values $>12.3 ; p<0.003$ ), effects of motor sequence boundaries $(F=5.02 ; p=$ $0.04)$, but no effect of task sequence boundaries $(F=1.55 ; p=0.23)$.

A similar pattern of phasic activations was observed in the ventral and dorsal right IPS regions exhibiting sustained activations. In both regions, we found marginal effects of motor increments $(F=$ $4.01 ; p=0.064)$, significant effects of task increments and motor sequence boundaries (both $F$ values $>7.1 ; p$ values $<0.018)$, but no effect of task sequence boundaries $(F<1.6 ; p>$ 0.22 ). Finally, post hoc analyses confirmed the dissociation between these two right IPL regions. The right dorsal IPS region exhibited sustained activations in both conditions (both $F$ values $>7.7$; $p$ values $<0.014$ ), whereas the right ventral IPS region exhibited sustained activations in the motor condition only (motor: $F>18.3, p<0.001$; task: $F<1.3, p>0.28$ ).

\section{The bilateral SMG/TPJ region}

As mentioned above, the bilateral SMG/TPJ region was the only brain region that exhibited significantly larger activations in endogenous than exogenous termination (Fig. 5) (main effect: $F=$ 8.7, $p=0.01$; interaction with condition, $F<1$; interaction with hemispheres: $F=3.0, p=0.1)$. Compared with motor and task increments, the region showed increased activations in motor and task sequence initiation $(F=5.4 ; p=0.035$; interaction with condition: $F=1.3, p=0.26)$ and endogenous termination $(F=$ 8.9; $p=0.009$; interaction with condition: $F=1.5, p=0.25)$. In contrast, no increased activations were observed in exogenous
Right dorsal IPS


Time (s)

Figure 3. Activations of parietal areas showing both sustained and phasic responses. Graphs show reconstruction of magnetic indicate SEs across subjects). For each time course, data points are adjusted; peristimulus MR signals are averaged in time bins of thained after subtracting the estimated contribution of other events computed from the multiple linear regression model. , Sustained effects in the left middle IPS, right dorsal IPS (purple regions in Fig. 2), and right ventral IPS (blue region). The orang ine indicates epoch-related activations during task sequence execution. The blue line indicates epoch-related activations during

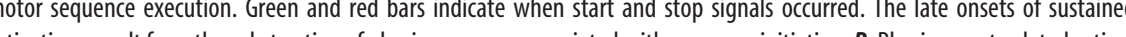
with squares indicate sequence boundary trials. $x$-axis origins are stimulus onsets.

termination compared with increments $(F=1.3 ; p=0.26$; interaction with condition, $F<1$ ). No differences were observed between the left and right region (interaction with hemisphere: all $F$ values $<2.6$; $p$ values $>0.12$ ).

\section{Discussion}

The present results first reveal that all posterior cortical activations related to the execution of prelearned behavioral sequences were confined to the inferior parietal cortex, confirming the prominent role of this region in processing behavioral sequences. Additionally, all these activations appear independent of the nature of processed stimuli (squares vs letters in the motor and task condition, respectively), because all reported regions exhibited phasic activations in both the motor and task conditions. Similarly, increased phasic activations at sequence boundaries are unlikely to reflect selective processing of the perceptual content of start and stop cues (colors). Indeed, regions showing such activations also exhibited increased activations in sequential increment compared with baseline trials or did not respond to specific 

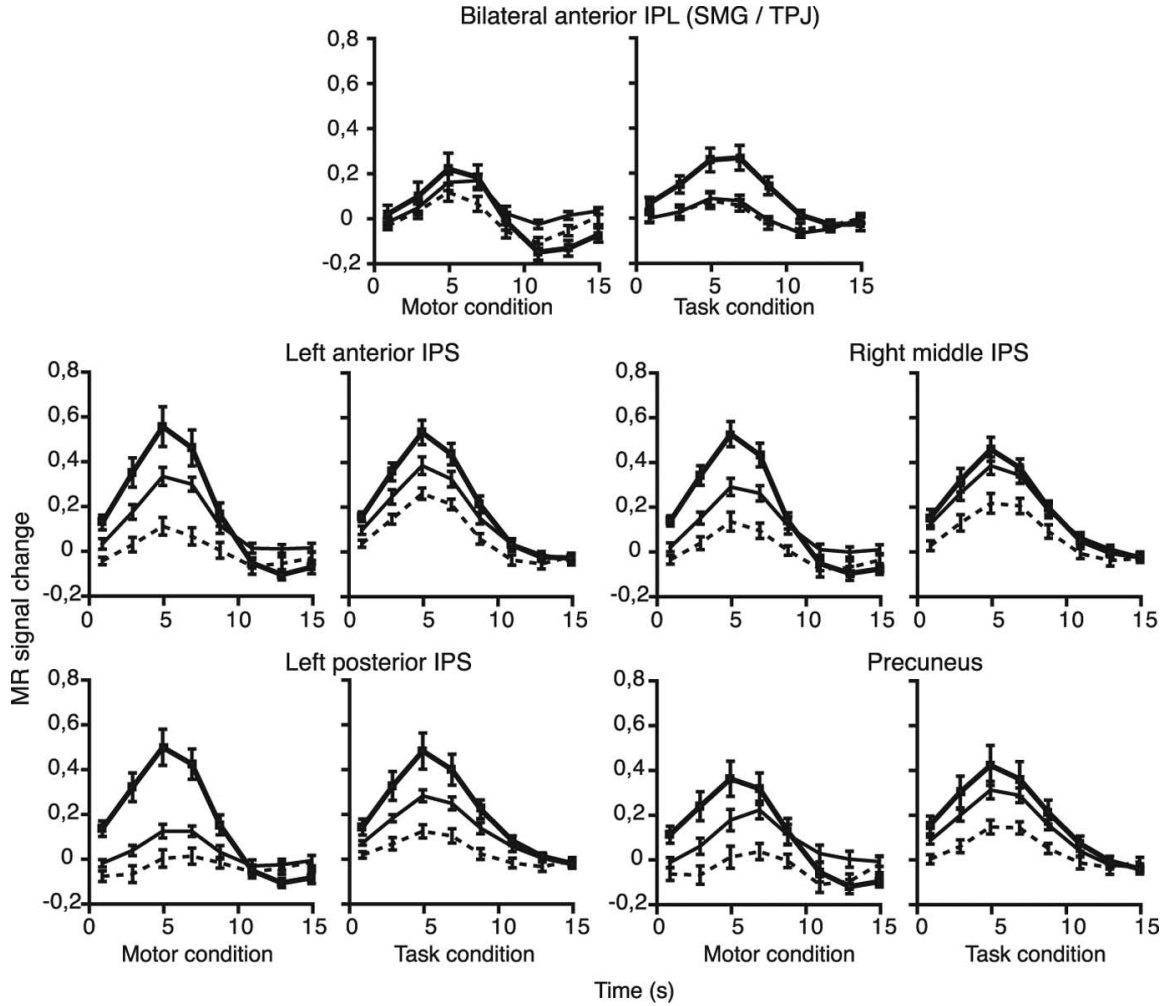

Figure 4. Activations of parietal regions showing phasic responses only. Graphs show reconstruction of magnetic resonance (MR) signal changes in the different conditions in specified regions averaged over clusters and subjects (error bars indicate SEs across subjects). For each time course, data points are adjusted; peristimulus MR signals are averaged in time bins of $2 \mathrm{~s}$ and obtained after subtracting the estimated contribution of other events computed from the multiple linear regression model. Event-related activations in the left anterior and posterior IPS (yellow and green regions in Fig. 2, respectively), right middle IPS and the precuneus (orange regions), and in bilateral SMG/TPJ regions (red regions) are shown. Dashed lines indicate baseline trials. Solid lines with no symbols indicate increment trials. Solid lines with squares indicate sequence boundary trials. $x$-axis origins are stimulus onsets. SMG/TPJ activations actually differed between endogenous and exogenous terminations (see Results and Fig. 5)

sequence boundary trials (task sequence boundaries in the right IPL and exogenous termination in the SMG/TPJ region). Thus, the data provide evidence that the reported inferior parietal activations are unrelated to the processing of the perceptual content of behavioral sequences.

The results especially reveal a single bilateral region located in the posterior IPS exhibiting sustained activations during the execution of both motor and task sequences. We did not find any other regions in the posterior cerebral cortex, and we did not previously identify any frontal regions showing the same activation pattern (Koechlin and Jubault, 2006). In the left IPS, the region also exhibited the same pattern of phasic responses during the execution of both motor and task sequences with especially increased activations at sequence boundaries. Such a joint pattern of sustained and phasic activations is expected in a region processing the abstract serial structure of action sequences (see Materials and Methods, Predictions), regardless of their hierarchical organization and sensorimotor content. According to this interpretation, sustained activations correspond to the maintenance of the serial structure and possibly the current position within this serial structure that guide sequential progression along behavioral sequences. Consistently, increased phasic activations at sequence boundaries simply reflect activation and inhibition of the entire sequence representation, whereas phasic activations associated with sequential increments correspond to updating the current position within such a representation.

Critically, the combination of sustained and phasic activations in this region rules out several alternative interpretations. Sustained activations cannot be simply explained by the increasing working-memory load required for maintaining the distinct elements that compose sequences, because this interpretation does not account for increased phasic activations observed at sequence termination. Phasic activations cannot be simply explained by switching between two successive, distinct elements composing behavioral sequences (motor responses and task sets), because phasic activations were also found in trials requiring subjects to repeat the preceding motor response or task set (sequence initiation trials). Furthermore, sustained activations are unlikely to reflect the maintenance of behavioral rules unrelated to the serial organization of motor and task sequences. Indeed, these behavioral rules, event contingencies, and performance durations were virtually identical in baseline and sequence execution periods. In both periods, subjects had to prepare the next action (movement or task sets), to respond to stimuli, to check color cues (for detecting start or stop cues), and so on. The only difference was that baseline and sequence periods involved sequences of identical versus distinct actions, respectively. This difference was associated with increased phasic activations in sequence increment compared with baseline trials. Finally, these left IPL activations might result from an increase in attentional demand during sequence execution (Cabeza and Nyberg, 2000; Cabeza et al., 2000; Corbetta et al., 2000; Culham and Kanwisher, 2001; Behrmann et al., 2004). This interpretation is unlikely, however, because attentional control engages both frontal and parietal regions (Cabeza and Nyberg, 2000), and, as mentioned above, no frontal regions showed sustained activations in both motor and task sequences.

In summary, our results provide evidence that the left middle IPS processes the abstract serial structure of behavioral plans regardless of their sensorimotor content and their hierarchical organization. This interpretation is consistent with previous results showing the involvement of the left IPS region in rehearsal of motor sequences versus rest condition (Catalan et al., 1998), of ordinal sequences versus temporal sequences (Bengtsson et al., 2004), and in encoding motor sequences at an abstract, effectorsindependent level (Grafton et al., 1998). Also, left IPS activations were consistently reported during execution of structured compared with unstructured sequences (Bor et al., 2003). This interpretation is also in accordance with the previously proposed hypothesis that the left parietal region is involved in shifting the focus of motor attention from one movement in a sequence to the next (Rushworth et al., 1997).

The right homolog of the left, middle IPS region also showed sustained activations during the execution of both motor and task sequences. However, the right IPS region showed a distinct pattern of phasic activations. This region responded to task increment and motor sequence boundaries but was not sensitive to 
Table 1. Activation peaks in the parietal cortex: epoch-related activations

\begin{tabular}{|c|c|c|c|c|}
\hline \multirow[b]{3}{*}{ Brain region } & \multicolumn{4}{|l|}{ Effect of sequence execution } \\
\hline & \multicolumn{2}{|l|}{ Motor condition } & \multicolumn{2}{|l|}{ Task condition } \\
\hline & Talairach coordinates (mm) & Max $t$ score (random effect) ${ }^{a}$ & Talairach coordinates (mm) & Max $t$ score (random effect) ${ }^{a}$ \\
\hline L middle IPS & $-40,-52,44$ & 4.5 & $-52,-48,44$ & 2.87 \\
\hline R dorsal IPS & $52,-44,44$ & 4.93 & $44,-44,48$ & 3.44 \\
\hline R ventral IPS & $52,-44,40$ & 4.54 & n.s. & \\
\hline
\end{tabular}

$L$, Left; $R$, right; $n$.s., nonsignificant contrasts even in post hoc analyses.

${ }^{a}$ Maximum (Max) $t$ scores (random-effect analysis) are for the statistical contrasts indicated in the table and described in Results.

Table 2. Activation peaks in the parietal cortex: event-related activations

\begin{tabular}{|c|c|c|c|c|c|c|c|c|}
\hline \multirow[b]{2}{*}{ Brain region } & \multicolumn{2}{|l|}{$\begin{array}{l}\text { Effect of increment } \\
\text { Motor condition }\end{array}$} & \multicolumn{2}{|l|}{ Task condition } & \multicolumn{2}{|c|}{$\begin{array}{l}\text { Effect of sequence boundary } \\
\text { Motor condition }\end{array}$} & \multicolumn{2}{|l|}{ Task condition } \\
\hline & $\begin{array}{l}\text { Talairach } \\
\text { coordinates }(\mathrm{mm})\end{array}$ & $\begin{array}{l}\text { Max } t \text { score } \\
\text { (random effect) }^{a}\end{array}$ & $\begin{array}{l}\text { Talairach } \\
\text { coordinates }(\mathrm{mm})\end{array}$ & $\begin{array}{l}\text { Maxtscore } \\
\text { (random effect) }^{a}\end{array}$ & $\begin{array}{l}\text { Talairach } \\
\text { coordinates }(\mathrm{mm})\end{array}$ & $\begin{array}{l}\text { Max tscore } \\
\text { (random effect) }^{a}\end{array}$ & $\begin{array}{l}\text { Talairach } \\
\text { coordinates }(\mathrm{mm})\end{array}$ & $\begin{array}{l}\text { Max t score } \\
\text { (random effect) }^{a}\end{array}$ \\
\hline L anterior IPS & $-44,-40,44$ & 5.04 & $-40,-48,44$ & 4.22 & $-40,-48,44$ & 4.12 & $-40,-48,40$ & 4.65 \\
\hline L posterior IPS & $-28,-72,44$ & 2.52 & $-28,-64,36$ & 5.97 & $-28,-72,36$ & 5.65 & $-32,-72,44$ & 3.33 \\
\hline R middle IPS & $32,-48,44$ & 5.09 & $36,-48,48$ & 5.35 & $36,-52,48$ & 5.11 & & n.s. \\
\hline L precuneus & $-20,-68,56$ & 3.35 & $-12,-68,52$ & 4.89 & $-20,-72,48$ & 3.72 & & n.s. \\
\hline $\mathrm{R}$ precuneus & $8,-72,52$ & 4.9 & $8,-72,52$ & 4.52 & & & & n.s. \\
\hline RSMG/TPJ & & n.s. & & n.s. & & sig. ${ }^{b}$ & $-56,-20,4$ & $3.45^{b}$ \\
\hline LSMG/TPJ & & n.s. & & n.s. & & sig. $^{b}$ & $52,-6,4$ & $3.06^{b}$ \\
\hline
\end{tabular}

$L$, Left; $R$, right; $n$.s., nonsignificant contrasts even in post hoc analyses.

${ }^{a}$ Maximum (Max) tscores (random-effect analysis) are for the statistical contrasts indicated in the table and described in Results.

${ }^{b}$ Post hoc significant (sig.) differences between endogenous and exogenous termination (see Results).

motor increments and task sequence boundaries. This activation pattern indicates that the region responded regardless of sequence boundaries and increments but only when subjects switched between simple action chunks [i.e., between either parallel sets of sensorimotor associations (task sets in the task condition) or serial sets of single acts (motor sequences in the motor condition)] (see Materials and Methods, Serial versus hierarchical organization of action sequences). Indeed, no phasic activations were observed when switching occurred only between single motor acts (motor increment) or when there was no or little task switching (task sequence boundaries included $72 \%$ trials with no task switching). Sustained activations are then likely to reflect the maintenance of selected simple action chunks in preparation of subsequent trials. In the motor condition, the entire sequence of motor acts is maintained from initiation to termination. In the task condition, a sensorimotor mapping is prepared in each increment trial and maintained up to the subsequent trial. This interpretation is consistent with a previous study showing right IPS activations associated with task-set preparation (Brass and von Cramon, 2004a). Moreover, contiguous to the right IPS region, a more ventral IPS region had the same pattern of phasic responses but exhibited sustained activations only during the execution of motor sequences. In motor compared with task sequences, motor responses could be additionally prepared between successive steps, suggesting that the right ventral IPS region is involved in further preparing single motor acts composing motor sequences. Consistently, we previously reported a similar activation pattern in the frontal regions involved in motor preparation, namely the supplementary motor area complex (Tanji and Shima, 1994; Koechlin and Jubault, 2006). Thus, our data provide evidence that the dorsal and ventral right IPL regions are engaged in preparing sensorimotor sets and motor acts, respectively, a function implicated but not specific to the execution of behavioral sequences (Asari et al., 2005; Shafritz et al., 2005). Finally, it is worth noting that we did not find any regions showing sustained activations in task sequences only, confirming that sustained activations are not segregated according to the hierarchical level of behavioral sequences.

Contiguous to these left and right IPS regions exhibiting sustained activations, additional IPS regions showed only phasic responses. These phasic responses as well as those found in the precuneus were also observed in contiguous sustained activations, except that all these regions additionally responded to motor increment. Importantly, brain regions that exhibited only phasic responses were also found in the frontal lobes (Koechlin and Jubault, 2006). More specifically, frontal regions responding to motor increment, as in these parietal regions, were found in the lateral premotor cortex only (BA 6). Posterior prefrontal regions (BA 44), in contrast, responded to motor sequence boundaries and task increment only (i.e., to boundaries of simple action chunks), whereas slightly more anterior prefrontal regions (BA 45) responded to task sequence boundaries only (i.e., boundaries of superordinate action chunks; see Materials and Methods, Serial versus hierarchical organization of action sequences). Thus, the lateral frontal cortex was proposed to form a functional system processing the hierarchical rather than serial structure of action plans (Koechlin and Jubault, 2006) with BAs 6, 44, and 45 involved in selecting single motor acts, simple and superordinate action chunks, respectively. In contrast, in parietal regions exhibiting only phasic responses, we did not find any evidence supporting such functional segregations based on the hierarchical levels of action plans, especially because all IPS regions and the precuneus responded to motor increments. In summary, this indicates that in contrast to prefrontal regions (i.e., BA 45), no parietal regions are specifically sensitive to recursive chunking of action segments (i.e., as in superordinate action chunks; see above).

An alternative hypothesis is that these IPS regions simply serve as input/output interfaces between the left IPS region, representing the serial structure of action plans, and the right IPS region, 


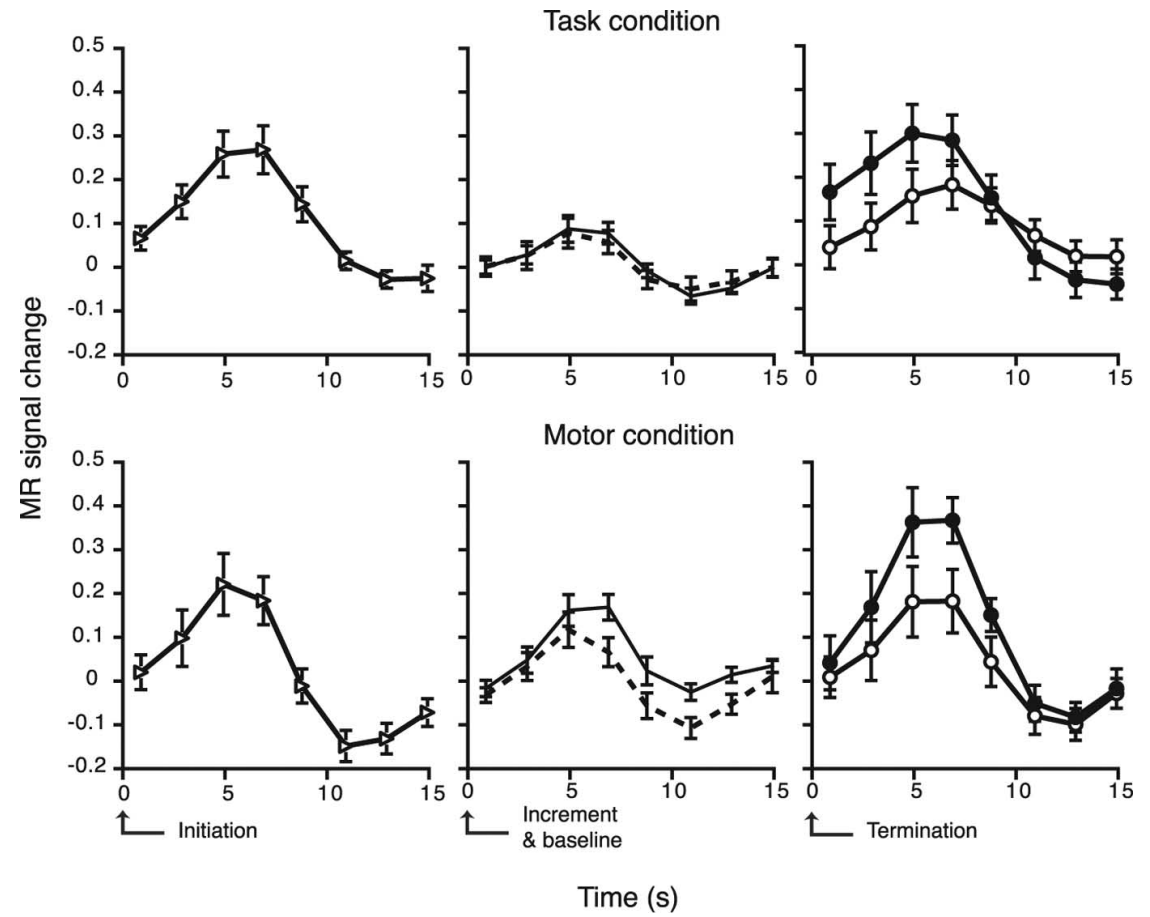

Figure 5. Phasic activations in the SMG/TPJ. Graphs show reconstruction of magnetic resonance (MR) signal changes in the different conditions in the SMG/TPJ averaged over clusters and subjects (error bars indicate SEs across subjects). For each time course, data points are adjusted; peristimulus MR signals are averaged in time bins of $2 \mathrm{~s}$ and obtained after subtracting the estimated contribution of other events computed from the multiple linear regression model. $x$-axis origins are stimulus onsets. Triangles indicate responses to sequence initiation. Dashed lines indicate responses in baseline trials. Solid lines indicate responses to sequence increment. Open and filled circles indicate responses to exogenous and endogenous termination, respectively.

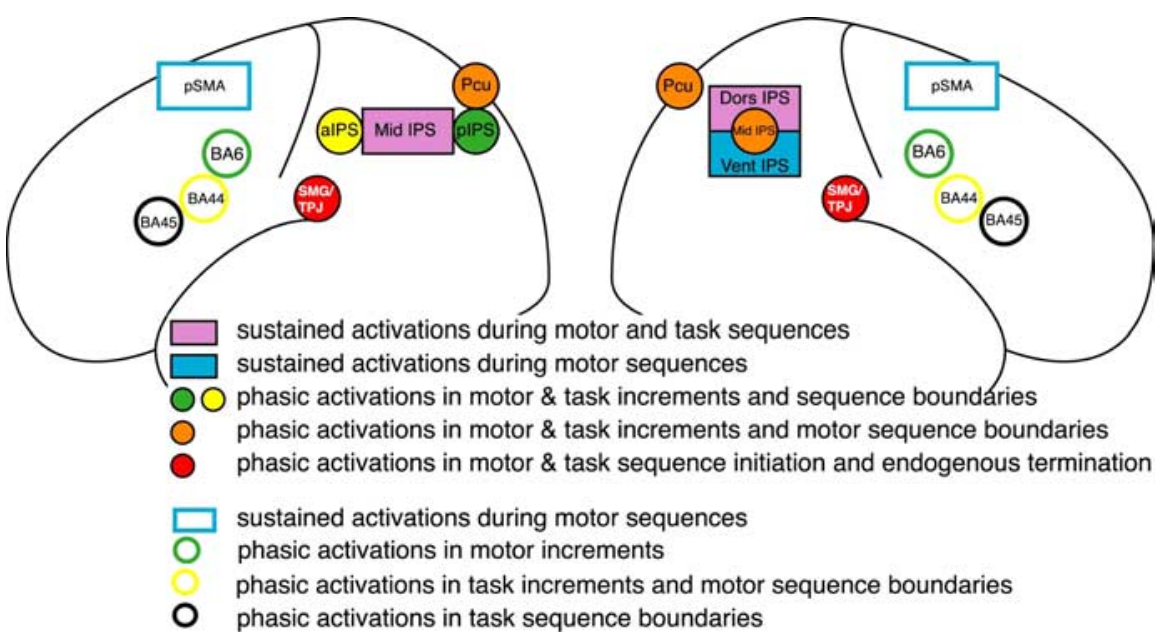

Figure 6. Schematic diagram of parietal and frontal activations observed in the protocol. Parietal activations are represented as solid circles and rectangles. Green and yellow colors indicate the interaction between motor increments and sequence boundaries effects in the anterior and posterior left IPS (green, larger effect of motor increment; yellow, larger effect of motor sequence boundaries). Frontal activations found in the same experimental protocol and reported by Koechlin and Jubault (2006) are shown as open circles and rectangles. Pcu, Precuneus; PSMA, presupplementary motor area.

preparing successive actions (see above). This idea is supported by the fact that these IPS regions with sustained responses showed similar phasic responses. In particular, during sequence execution, the precuneus may be involved in coupling the serial-order and preparatory representations described above in the left and right IPS. Consistently, systematic connections between the precuneus and adjacent parietal areas including the IPL have been reported previously (Leichnetz, 2001), as well as the involvement of the precuneus in processing sequential complexity (Sadato et al., 1996) and in highly integrated functions irreducible to simple visuospatial processing (Cavanna and Trimble, 2006). The IPS regions exhibiting phasic responses may also serve as attentional gates between the IPL and the frontal cortex coupling serial-order and preparatory representations in the IPL with the hierarchical organization of action in the frontal cortex (Bunge, 2004). This hypothesis is supported by the differential responses to motor increments found in the posterior and anterior left IPS regions that somewhat resembles the functional segregation previously observed between premotor and prefrontal regions (see above) (Koechlin and Jubault, 2006).

Finally, our results reveal a bilateral region located in the inferior part of the SMG/TPJ that responded only to initiation and endogenous termination of both motor and task sequences. Again, in these regions there were no significant differences between motor and task sequences. Moreover, this pattern of responses is unique and was found in no other brain regions. Unlike exogenous termination, endogenous termination corresponded to stop signals occurring at the end state of learned sequences. Similarly, initiation trials corresponded to the start state of learned sequences, so that the region responded only to the "true" boundaries of memorized sequences. Thus, the SMG/ TPJ region appears to be associated with the long-term memory of behavioral sequences. In the left hemisphere, this brain sector has also been found in processing linguistic sequences (Aboitiz and Garcia, 1997; Sakai et al., 2001). However, SMG/ TPJ activations reported here are unlikely to reflect any covert verbal processing, because activations were bilateral and associated with sequence boundaries only. Instead, we interpret such phasic responses as reflecting retrieval of long-term representations of learned behavioral sequences when start cues occur and intrinsic inhibition of ongoing behavioral sequences when memorized end states are reached.

In conclusion, our results suggest a system of parietal regions located in the IPS and involved in the serial organization of behavior (Fig. 6). In this system, the left IPS processes the abstract serial structure of action plans that guides and keeps track of sequential progression within behavioral plans, regardless of their hierarchical structure. The right IPS is involved in successively preparing simple action chunks (dorsal IPS) and, if possible, single motor acts (ventral IPS) that compose such behavioral plans. This parietal system strongly differs from the frontal system controlling action selection with respect to the hierarchical rather than serial structure of behavioral 
plans (Koechlin and Jubault, 2006). Thus, our results suggest a major functional segregation within the frontoparietal network mediating action planning (Bunge et al., 2002; Rushworth et al., 2003; Brass and von Cramon, 2004b; Brass et al., 2005), with the frontal and parietal sector involved in processing the hierarchical and serial structure of action plans, respectively.

\section{References}

Aboitiz F, Garcia VR (1997) The evolutionary origin of the language areas in the human brain. A neuroanatomical perspective. Brain Res Brain Res Rev 25:381-396.

Andersen RA, Snyder LH, Bradley DC, Xing J (1997) Multimodal representation of space in the posterior parietal cortex and its use in planning movements. Annu Rev Neurosci 20:303-330.

Asari T, Konishi S, Jimura K, Miyashita Y (2005) Multiple components of lateral posterior parietal activation associated with cognitive set shifting. NeuroImage 26:694-702.

Baker SC, Rogers RD, Owen AM, Frith CD, Dolan RJ, Frackowiak RS, Robbins TW (1996) Neural systems engaged by planning: a PET study of the Tower of London task. Neuropsychologia 34:515-526.

Barash S (2003) Paradoxical activities: insight into the relationship of parietal and prefrontal cortices. Trends Neurosci 26:582-589.

Behrmann M, Geng JJ, Shomstein S (2004) Parietal cortex and attention. Curr Opin Neurobiol 14:212-217.

Bengtsson SL, Ehrsson HH, Forssberg H, Ullen F (2004) Dissociating brain regions controlling the temporal and ordinal structure of learned movement sequences. Eur J Neurosci 19:2591-2602.

Bengtsson SL, Ehrsson HH, Forssberg H, Ullen F (2005) Effectorindependent voluntary timing: behavioural and neuroimaging evidence. Eur J Neurosci 22:3255-3265.

Bor D, Duncan J, Wiseman RJ, Owen AM (2003) Encoding strategies dissociate prefrontal activity from working memory demand. Neuron 37:361-367.

Brass M, von Cramon DY (2004a) Decomposing components of task preparation with functional magnetic resonance imaging. J Cogn Neurosci 16:609-620.

Brass M, von Cramon DY (2004b) Selection for cognitive control: a functional magnetic resonance imaging study on the selection of task-relevant information. J Neurosci 24:8847-8852.

Brass M, Ullsperger M, Knoesche TR, von Cramon DY, Phillips NA (2005) Who comes first? The role of the prefrontal and parietal cortex in cognitive control. J Cogn Neurosci 17:1367-1375.

Bunge SA (2004) How we use rules to select actions: a review of evidence from cognitive neuroscience. Cogn Affect Behav Neurosci 4:564-579.

Bunge SA, Hazeltine E, Scanlon MD, Rosen AC, Gabrieli JD (2002) Dissociable contributions of prefrontal and parietal cortices to response selection. NeuroImage 17:1562-1571.

Cabeza R, Nyberg L (2000) Imaging cognition II: An empirical review of 275 PET and fMRI studies. J Cogn Neurosci 12:1-47.

Cabeza R, Anderson ND, Houle S, Mangels JA, Nyberg L (2000) Age-related differences in neural activity during item and temporal-order memory retrieval: a positron emission tomography study. J Cogn Neurosci 12:197-206.

Catalan MJ, Honda M, Weeks RA, Cohen LG, Hallett M (1998) The func- tional neuroanatomy of simple and complex sequential finger movements: a PET study. Brain 121:253-264.

Cavanna AE, Trimble MR (2006) The precuneus: a review of its functional anatomy and behavioural correlates. Brain 129:564-583.

Cohen YE, Andersen RA (2002) A common reference frame for movement plans in the posterior parietal cortex. Nat Rev Neurosci 3:553-562.

Collette F, Olivier L, Van der Linden M, Laureys S, Delfiore G, Luxen A, Salmon E (2005) Involvement of both prefrontal and inferior parietal cortex in dual-task performance. Brain Res Cogn Brain Res 24:237-251.

Corbetta M, Kincade JM, Ollinger JM, McAvoy MP, Shulman GL (2000) Voluntary orienting is dissociated from target detection in human posterior parietal cortex. Nat Neurosci 3:292-297.

Culham JC, Kanwisher NG (2001) Neuroimaging of cognitive functions in human parietal cortex. Curr Opin Neurobiol 11:157-163.

Deiber MP, Ibanez V, Sadato N, Hallett M (1996) Cerebral structures participating in motor preparation in humans: a positron emission tomography study. J Neurophysiol 75:233-247.

Fincham JM, Carter CS, van Veen V, Stenger VA, Anderson JR (2002) Neural mechanisms of planning: a computational analysis using event-related fMRI. Proc Natl Acad Sci USA 99:3346-3351.

Fuster JM (1989) The prefrontal cortex, Ed 2. New York: Raven.

Grafton ST, Hazeltine E, Ivry RB (1998) Abstract and effector-specific representations of motor sequences identified with PET. J Neurosci 18:9420-9428.

Koechlin E, Jubault T (2006) Broca's area and the hierarchical organization of human behavior. Neuron 50:963-974.

Koechlin E, Danek A, Burnod Y, Grafman J (2002) Medial prefrontal and subcortical mechanisms underlying the acquisition of motor and cognitive action sequences in humans. Neuron 35:371-381.

Leichnetz GR (2001) Connections of the medial posterior parietal cortex (area 7m) in the monkey. Anat Rec 263:215-236.

Rushworth MF, Nixon PD, Renowden S, Wade DT, Passingham RE (1997) The left parietal cortex and motor attention. Neuropsychologia 35:1261-1273.

Rushworth MF, Johansen-Berg H, Gobel SM, Devlin JT (2003) The left parietal and premotor cortices: motor attention and selection. NeuroImage 20 [Suppl 1]:S89-S100.

Sadato N, Campbell G, Ibanez V, Deiber M, Hallett M (1996) Complexity affects regional cerebral blood flow change during sequential finger movements. J Neurosci 16:2691-2700.

Sakai K, Ramnani N, Passingham RE (2002) Learning of sequences of finger movements and timing: frontal lobe and action-oriented representation. J Neurophysiol 88:2035-2046.

Sakai KL, Hashimoto R, Homae F (2001) Sentence processing in the cerebral cortex. Neurosci Res 39:1-10.

Shafritz KM, Kartheiser P, Belger A (2005) Dissociation of neural systems mediating shifts in behavioral response and cognitive set. NeuroImage 25:600-606.

Talairach J, Tournoux P (1988) Co-planar stereotaxic atlas of the human brain. New York: Thieme Medical Publishers.

Tanji J, Shima K (1994) Role for supplementary motor area cells in planning several movements ahead. Nature 371:413-416.

Wu T, Kansaku K, Hallett M (2004) How self-initiated memorized movements become automatic: a functional MRI study. J Neurophysiol 91: $1690-1698$. 